\title{
ON FIRST-PASSAGE-TIME AND TRANSITION DENSITIES FOR STRONGLY SYMMETRIC DIFFUSION PROCESSES
}

\author{
A. DI CRESCENZO ${ }^{1)}$, V. GIORNO ${ }^{2)}$, A. G. NOBILE ${ }^{2)}$ \\ AND L. M. RICCIARDI ${ }^{1)}$
}

\section{Introduction}

One dimensional diffusion processes have been increasingly invoked to model a variety of biological, physical and engineering systems subject to random fluctuations (cf., for instance, Blake, I. F. and Lindsey, W. C. [2], Abrahams, J. [1], Giorno, V. et al. [10] and references therein). However, usually the knowledge of the 'free' transition probability density function (pdf) is not sufficient; one is thus led to the more complicated task of determining transition functions in the presence of preassigned absorbing boundaries, or first-passage-time densities for time-dependent boundaries (see, for instance, Daniels, H. E. [6], [7], Giorno, V. et al. [10]). Such densities are known analytically only in some special instances so that numerical methods have to be implemented in general (cf., for instance, Buonocore, A. et al. [3], [4], Giorno, V. et al. [11]). The analytical approach becomes particularly effective when the diffusion process exhibits some special features, such as the symmetry of its transition pdf. For instance, in [10] special symmetry conditions on the transition pdf of one-dimensional time-homogeneous diffusion process with natural boundaries are investigated to derive closed form results concerning the transition pdf's and the first-passage-time pdf for particular time-dependent boundaries. On the other hand, by using the method of images, in [6] Daniels has obtained a closed form expression for the transition pdf of the standard Wiener process in the presence of a particular time-dependent absorbing boundary. It is interesting to remark that such density cannot be obtained via the methods described in [10], even though the considered process exhibits the kind of symmetry discussed therein.

The purpose of the present paper is to make use of a variant of the method of

Received May 26, 1993.

Revised October 26, 1994. 
images in order to disclose new transition pdf's in the presence of time-dependent boundaries, as well as new first-passage-time pdf's, for a particular class of symmetric processes that also include Daniel's mentioned result for the standard Wiener process.

In the remaining part of this Section we shall establish the notation and state some preliminary results.

Let $\left\{X(t) ; t \geq t_{0}, t_{0} \in \mathbf{R}\right\}$ be a time-homogeneous diffusion process defined over the interval $I \equiv\left(\nu_{1}, \nu_{2}\right)$, with $\nu_{1}$ and $\nu_{2}$ natural boundaries and let $A_{1}(x)$ and $A_{2}(x)$ be the drift and the infinitesimal variance of $X(t)$, respectively. Following Feller, W. [8], [9], we assume that for all $x \in I, A_{1}(x), A_{2}(x)$ and $A_{2}^{\prime}(x)$ are defined and continuous functions, with $A_{2}(x)>0$. Let $\tau \geq t_{0}$ be an arbitrary instant. For all $t>\tau$ and $x, y \in I$ let us denote by

$$
f(x, t \mid y, \tau)=\frac{\partial}{\partial x} P\{X(t)<x \mid X(\tau)=y\}
$$

the transition pdf. As proved in [8], since $\nu_{1}$ and $\nu_{2}$ have been assumed to be natural boundaries, the transition pdf $f(x, t \mid y, \tau)$ is the unique solution of the Fokker-Planck equation

$$
\frac{\partial}{\partial t} f(x, t \mid y, \tau)=-\frac{\partial}{\partial x}\left[A_{1}(x) f(x, t \mid y, \tau)\right]+\frac{1}{2} \frac{\partial^{2}}{\partial x^{2}}\left[A_{2}(x) f(x, t \mid y, \tau)\right]
$$

with the initial delta condition:

$$
\lim _{t \downarrow \tau} f(x, t \mid y, \tau)=\delta(x-y) .
$$

Throughout this paper we shall deal with a subclass of the symmetric processes introduced and analyzed in [10].

Lemma 1.1. Let $\phi(x, t)$ be a continuous function of $x$ mapping $I$ onto itself for all $t \geq \tau$ such that $\partial \psi(x, t) / \partial x, \partial^{2} \psi(x, t) / \partial x^{2}, \partial^{3} \psi(x, t) / \partial x^{3}, \partial \psi(x, t) / \partial t$, $\partial^{2} \psi(x, t) / \partial x \partial t$ exist, with $\partial \phi(x, t) / \partial x<0$ for all $t \geq \tau$ and $x \in I$. Furthermore, let $r(t) \in C_{2}[\tau,+\infty)$ be such that

$$
\phi[r(t), t]=r(t),
$$

and let

$$
\varphi(x, t)=\left|\frac{\partial \psi(x, t)}{\partial x}\right|^{1 / 2} \exp \left\{-\int_{x}^{\psi(x, t)} d z \frac{A_{1}(z)}{A_{2}(z)}+\frac{2 r^{\prime}(t)}{\sqrt{A_{2}[r(t)]}} W[x, r(t)]\right\}
$$


with

$$
W(a, b)=\int_{a}^{b} \frac{d z}{\sqrt{A_{2}(z)}}
$$

Then, for all $x, y \in I$ and $t \geq \tau$

$$
f(x, t \mid y, \tau)=\frac{\varphi(x, t)}{\varphi(y, \tau)}\left|\frac{\partial \phi(x, t)}{\partial x}\right| f[\phi(x, t), t \mid \phi(y, \tau), \tau]
$$

iff

$$
A_{2}[\psi(x, t)]=A_{2}(x)\left[\frac{\partial \psi(x, t)}{\partial x}\right]^{2}
$$

(1.8b) $\frac{\partial \varphi(x, t)}{\partial t}+A_{1}(x) \frac{\partial \varphi(x, t)}{\partial x}+\frac{A_{2}(x)}{2} \frac{\partial^{2} \varphi(x, t)}{\partial x^{2}}-\frac{A_{2}(x)}{\varphi(x, t)}\left(\frac{\partial \varphi(x, t)}{\partial x}\right)^{2}=0$.

Proof. It follows by a procedure similar to that exploited for Theorem 3.1 of [10]. Note that here the additional assumption is made that $\phi$ is a strictly decreasing function of $x$ satisfying (1.4). Therefore, expression (1.5) of $\varphi$ is now obtained in place of the corresponding expression given in [10].

It should be stressed that the knowledge of the infinitesimal moments $A_{1}(x)$ and $A_{2}(x)$ of the process allows one to establish via (1.4), (1.5) and (1.8) whether functions $\varphi$ and $\phi$ exist such that (1.7) holds. It is worth pointing out that for any $x<r(t)[x>r(t)]$ one has $\psi(x, t)>r(t)[\psi(x, t)<r(t)]$. It is thus natural to call $r(t)$ a symmetry curve with respect to the symmetry function $\phi(x, y)$. We remark that, since $\phi(x, t)$ is a monotonic decreasing function of $x$ satisfying (1.4), relation (1.8a) is equivalent to

$$
W[x, r(t)]=W[r(t), \phi(x, t)] .
$$

Hence, for all $x \in I$ and $t \geq \tau$ the symmetry function $\phi(x, t)$ is such that

$$
\phi[\phi(x, t), t]=x
$$

The meaning of $\varphi$ is illustrated by remarking that for a given symmetry curve $r(t)$ and for all $t \geq \tau$ from (1.8a) and (1.5) one has $\left.\frac{\partial \phi(x, t)}{\partial x}\right|_{x=r(t)}=-1$ and $\varphi[r(t), t]=1$. Hence, for all $y \in I$ and $t>\tau$ from (1.7) one obtains $\varphi(y, \tau)=$ $f[r(t), t \mid \phi(y, \tau), \tau] / f[r(t), t \mid y, \tau]$. The function $\varphi$ thus expresses a ratio of transition densities that becomes independent of the current time $t$ by virtue of the 
role played by the symmetry curve $r$ and the symmetry function $\phi$. In the sequel, under the assumptions of Lemma 1.1, any function $r$ such that (1.4) holds will be called a symmetry curve, while $\phi$ and $\varphi$ will be denoted as the corresponding symmetry functions.

We remark that, under the assumptions of Lemma 1.1, for all $x, y \in I$ and $t>\tau$ the function $\frac{1}{f} \frac{\partial f}{\partial x}$ satisfies the following relation:

$$
\frac{1}{f(x, t \mid y, \tau)} \frac{\partial f(x, t \mid y, \tau)}{\partial x}
$$

$$
\begin{aligned}
= & \frac{\partial \phi(x, t)}{\partial x}\left\{\frac{1}{f[z, t \mid \phi(y, \tau), \tau]} \frac{\partial f[z, t \mid \phi(y, \tau), \tau]}{\partial z}\right\}_{z=\psi(x, t)}+\frac{3}{2}\left(\frac{\partial \phi(x, t)}{\partial x}\right)^{-1} \\
& \times \frac{\partial^{2} \phi(x, t)}{\partial x^{2}}-\left(\frac{\partial \psi(x, t)}{\partial x}\right)^{-1} \frac{A_{1}[\psi(x, t)]}{A_{2}(x)}+\frac{A_{1}(x)}{A_{2}(x)}-\frac{2 r^{\prime}(t)}{\sqrt{A_{2}(x)} \sqrt{A_{2}[r(t)]}} .
\end{aligned}
$$

Lemma 1.1 relates the function $f(x, t \mid y, \tau)$ to the function $f[\psi(x, t)$, $t \mid \phi(y, \tau), \tau]$. In general no relation exists between $f(x, t \mid y, \tau)$. and $f[\psi(x, t)$, $t \mid y, \tau]$. However, the following theorem shows that such a relation does exist if and only if $\frac{1}{f} \frac{\partial f}{\partial x}$ has a particular form.

THEOREM 1.1. For all $t>\tau$ let $Q(t, \tau)$ be a continuous positive function such that $\partial Q / \partial t$ exists and

$$
\lim _{t \downarrow \tau} Q(t, \tau)=0
$$

Then, under the assumptions of Lemma 1.1,

$$
\begin{gathered}
\varphi(x, t)\left|\frac{\partial \phi(x, t)}{\partial x}\right| f[\phi(x, t), t \mid y, \tau]=f(x, t \mid y, \tau) \\
\times \exp \left\{-\frac{2}{Q(t, \tau)} W[x, r(t)] W[y, r(\tau)]\right\}
\end{gathered}
$$

iff

$$
\begin{gathered}
\frac{1}{f(x, t \mid y, \tau)} \frac{\partial f(x, t \mid y, \tau)}{\partial x}=\frac{A_{1}(x)}{A_{2}(x)}-\frac{3}{4} \frac{A_{2}^{\prime}(x)}{A_{2}(x)}+\frac{1}{\sqrt{A_{2}(x)}} \\
\times\left\{\frac{1}{Q(t, \tau)} \frac{\partial Q(t, \tau)}{\partial t} W[x, r(t)]-\frac{1}{Q(t, \tau)} W[y, r(\tau)]-\frac{r^{\prime}(t)}{\sqrt{A_{2}[r(t)]}}\right\} .
\end{gathered}
$$


Proof. First of all we check that (1.14) satisfies relation (1.11). Since $\phi(x, t)$ is a monotonic decreasing function of $x$, from (1.8a) we have:

$$
\left.\frac{d A_{2}(z)}{d z}\right|_{z=\psi(x, t)}=A_{2}^{\prime}(x) \frac{\partial \psi(x, t)}{\partial x}+2 A_{2}(x) \frac{\partial^{2} \psi(x, t)}{\partial x^{2}} .
$$

Making then use of (1.8a), (1.9) and (1.15), from (1.14) one obtains:

$$
\left\{\frac{1}{f[z, t \mid \phi(y, \tau), \tau]} \frac{\partial f[z, t \mid \psi(y, \tau), \tau]}{\partial z}\right\}_{z=\psi(x, t)}
$$

$$
\begin{aligned}
=\frac{A_{1}[\psi(x, t)]}{A_{2}(x)}\left(\frac{\partial \psi(x, t)}{\partial x}\right)^{-2} & -\frac{3}{4}\left(\frac{\partial \psi(x, t)}{\partial x}\right)^{-1} \frac{A_{2}^{\prime}(x)}{A_{2}(x)}-\frac{3}{2}\left(\frac{\partial \psi(x, t)}{\partial x}\right)^{-2} \frac{\partial^{2} \psi(x, t)}{\partial x^{2}} \\
& +\frac{1}{\sqrt{A_{2}(x)}}\left(\frac{\partial \psi(x, t)}{\partial x}\right)^{-1}\left\{\frac{1}{Q(t, \tau)} \frac{\partial Q(t, \tau)}{\partial t} W[x, r(t)]\right. \\
& \left.-\frac{1}{Q(t, \tau)} W[y, r(\tau)]+\frac{r^{\prime}(t)}{\sqrt{A_{2}[r(t)]}}\right\} .
\end{aligned}
$$

One easily sees that (1.14) and (1.16) satisfy (1.11). Hence, (1.14) is in agreement with the assumptions of Lemma 1.1. We now prove that (1.13) implies (1.14). To this end we notice that due to (1.7), the left-hand-side of (1.13) satisfies the Fokker-Planck equation (1.2). Hence, we set

$$
H(x, t \mid y, \tau)=\exp \left\{-\frac{2}{Q(t, \tau)} W[x, r(t)] W[y, r(\tau)]\right\}
$$

and impose that also the right-hand-side of (1.13), i.e. the product $f(x, t \mid y, \tau)$ $H(x, t \mid y, \tau)$, for $t>\tau$ satisfies the Fokker-Planck equation

$$
\frac{\partial}{\partial t}(f H)+\frac{\partial}{\partial x}\left[A_{1}(x) f H\right]-\frac{1}{2} \frac{\partial^{2}}{\partial x^{2}}\left[A_{2}(x) f H\right]=0 .
$$

Since $\left(\frac{\partial H}{\partial x}\right)^{-1} \frac{\partial H}{\partial t}$ and $\left(\frac{\partial H}{\partial t}\right)^{-1} \frac{\partial^{2} H}{\partial x^{2}}$ exist for all $x, y \in I$ and $t>\tau$, making use of (1.2), equation (1.18) becomes:

$$
\frac{1}{f} \frac{\partial f}{\partial x}=\frac{A_{1}(x)}{A_{2}(x)}-\frac{A_{2}^{\prime}(x)}{A_{2}(x)}+\frac{1}{A_{2}(x)}\left(\frac{\partial H}{\partial x}\right)^{-1} \frac{\partial H}{\partial t}-\frac{1}{2}\left(\frac{\partial H}{\partial x}\right)^{-1} \frac{\partial^{2} H}{\partial x^{2}}
$$

Recalling definitions (1.17), from (1.19) we immediately obtain (1.14). This proves the necessary part of the theorem. To prove the sufficiency we integrate both sides of $(1.14)$ in the interval $(x, \psi(x, t))$. Then, 
(1.20)

$$
\begin{aligned}
& f[\phi(x, t), t \mid y, \tau] \\
& =f(x, t \mid y, \tau) \exp \left\{\int_{x}^{\psi(x, t)} d z \frac{A_{1}(z)}{A_{2}(z)}-\frac{3}{4} \ln \frac{A_{2}[\psi(x, t)]}{A_{2}(x)}\right. \\
& +\frac{1}{Q(t, \tau)} \frac{\partial Q(t, \tau)}{\partial t} \int_{x}^{\psi(x, t)} d z \frac{W[z, r(t)]}{\sqrt{A_{2}(z)}}-\frac{1}{Q(t, \tau)} W[x, \phi(x, t)] \\
& \left.\quad \times W[y, r(\tau)]-\frac{r^{\prime}(t)}{\sqrt{A_{2}[r(t)]}} W[x, \phi(x, t)]\right\} .
\end{aligned}
$$

Due to $(1.8 \mathrm{a})$ we also have:

$$
W[x, \phi(x, t)]=2 W[x, r(t)], \quad \int_{x}^{\psi(x, t)} d z \frac{W[z, r(t)]}{\sqrt{A_{2}(z)}}=0 .
$$

Hence, making use of (1.21) equation (1.20) becomes:

$$
\begin{aligned}
f[\psi(x, t), t \mid y, \tau]= & f(x, t \mid y, \tau)\left|\frac{\partial \psi(x, t)}{\partial x}\right|^{-3 / 2} \\
& \times \exp \left\{\int_{x}^{\psi(x, t)} d z \frac{A_{1}(z)}{A_{2}(z)}-\frac{2 r^{\prime}(t)}{\sqrt{A_{2}[r(t)]}} W[x, r(t)]\right\} \\
& \times \exp \left\{-\frac{2}{Q(t, \tau)} W[x, r(t)] W[y, r(\tau)]\right\} .
\end{aligned}
$$

Recalling Definition (1.5), from (1.22) Relation (1.13) follows. Note that our assumptions on the function $Q(t, \tau)$ insure the validity of $(1.13)$ as $t$ tends to $\tau$. $\square$

The nature of the function $Q(t, \tau)$ that appears in (1.13) is elucidated by the following corollary.

COROLlary 1.1. Let $r_{i}(t)(i=1,2)$ be two arbitrary symmetry curves such that for all $t \geq \tau$ one has $r_{1}(t) \neq r_{2}(t)$ and let $Q_{i}(t, \tau)(i=1,2)$ be the corresponding functions in equation (1.13). Then, under the assumptions of Theorem 1.1, one has:

$$
Q_{1}(t, \tau)=Q_{2}(t, \tau)=D(t) D(\tau) \int_{\tau}^{t} \frac{d \vartheta}{D^{2}(\vartheta)}
$$

where we have set

$$
D(t)=W\left[r_{1}(t), r_{2}(t)\right]
$$

Proof. From (1.14) one has: 


$$
\left[\frac{1}{f(x, t \mid y, \tau)} \frac{\partial f(x, t \mid y, \tau)}{\partial x}-\frac{A_{1}(x)}{A_{2}(x)}+\frac{3}{4} \frac{A_{2}^{\prime}(x)}{A_{2}(x)}\right] \sqrt{A_{2}(x)}
$$

$=\frac{1}{Q_{i}(t, \tau)} \frac{\partial Q_{i}(t, \tau)}{\partial t} W\left[x, r_{i}(t)\right]-\frac{1}{Q_{i}(t, \tau)} W\left[y, r_{i}(\tau)\right]-\frac{r_{i}^{\prime}(t)}{\sqrt{A_{2}\left[r_{\imath}(t)\right]}} \quad(i=1,2)$.

Then, from (1.25) for $i=1,2$ one obtains:

$$
\begin{aligned}
& {\left[\frac{1}{Q_{1}(t, \tau)} \frac{\partial Q_{1}(t, \tau)}{\partial t}-\frac{1}{Q_{2}(t, \tau)} \frac{\partial Q_{2}(t, \tau)}{\partial t}\right] W\left[x, r_{1}(t)\right]} \\
& \quad-\left[\frac{1}{Q_{1}(t, \tau)}-\frac{1}{Q_{2}(t, \tau)}\right] W\left[y, r_{1}(\tau)\right] \\
& =\frac{1}{Q_{2}(t, \tau)} \frac{\partial Q_{2}(t, \tau)}{\partial t} D(t)-\frac{1}{Q_{2}(t, \tau)} D(\tau)-D^{\prime}(t)
\end{aligned}
$$

where $D(t)$ is defined in (1.24). In order for (1.26) to hold for all $x, y \in I$ and $t>\tau$, it must be $Q_{1}(t, \tau)=Q_{2}(t, \tau)$. Furthermore, Equation (1.26) becomes:

$$
\frac{\partial Q_{2}(t, \tau)}{\partial t}-\frac{D^{\prime}(t)}{D(t)} Q_{2}(t, \tau)-\frac{D(\tau)}{D(t)}=0
$$

Recalling (1.12), from (1.27) the expression (1.23) then finally follows.

Corollary 1.1 shows that for any two symmetry curves $r_{1}(t)$ and $r_{2}(t)$ such that $r_{1}(t) \neq r_{2}(t)$ for all $t \geq \tau$, the function $Q_{1}(t, \tau)$ related to $r_{1}(t)$ is known (cf. (1.23)) and it coincides with the function $Q_{2}(t, \tau)$ related to $r_{2}(t)$.

In for instance, $A_{2}(x)=\sigma^{2}$ and $r_{1}(t)=a t+b, r_{2}(t)=a^{*} t+b^{*}$ with $r_{1}(t)$ $\neq r_{2}(t)$ for all $t \geq \tau$, then from (1.23) and (1.24) we obtain $Q_{1}(t, \tau)=Q_{2}(t, \tau)=$ $t-\tau$.

Definition 1.1. A diffusion process will be said to be strongly symmetric iff relations (1.7) and (1.13) hold under the assumptions of validity of Theorem 1.1.

In the sequel we shall confine our attention exclusively to strongly symmetric processes.

\section{One absorbing boundary}

Let $y(t), u(t)$ and $v(t)$ be symmetry curves such that $y(t)<u(t)<v(t)$ $[y(t)>u(t)>v(t)]$ for all $t \geq \tau$, where $\tau \geq t_{0}$ is a fixed instant. We denote by $\phi_{1}(x, t)$ and $\varphi_{1}(x, t)$ the symmetry functions corresponding to $u(t)$ and by $\phi_{2}(x, t)$ 
and $\varphi_{2}(x, t)$ the symmetry functions for $v(t)$. Furthermore we assume that

$$
v(t)=\phi_{1}[y(t), t] .
$$

Such an assumption implies that the symmetry curve $v(t)$ is the "image" of the symmetry curve $y(t)$ in the "mirror" $u(t)$ via the symmetry function $\phi_{1}$. If, for instance, for all $t \geq \tau$ ( $\tau$ fixed) one has $y(t)<u(t)<v(t)$, the conditions $y(t)<x<u(t)$ amount to requiring $\phi_{1}[y(t), t]>\phi_{1}(x, t)>\phi_{1}[u(t), t]$, i.e. $u(t)<\phi_{1}(x, t)<v(t)$.

Let us now assume $P\{X(\tau)=y(\tau)\}=1$ and let $S(t ; \tau)$ be a continuous function of $t$ taking values in $I$ for all $t \geq \tau$. For all $t \geq \tau$ and $x \in I$ we set:

$$
A(x, t \mid y(\tau), \tau)=\left\{\begin{array}{r}
\left(x<S(t ; \tau) ; y(\tau)<\lim _{t \downarrow \tau} S(t ; \tau)\right), \\
P\{X(t)<x ; X(\vartheta)>S(\vartheta ; \tau) \forall \vartheta \in(\tau, t) \mid X(\tau)=y(\tau)\} \\
\left(x>S(t ; \tau) ; y(\tau)>\lim _{t \downarrow \tau} S(t ; \tau)\right)
\end{array}\right.
$$

and

$$
\alpha(x, t \mid y(\tau), \tau)=\frac{\partial}{\partial x} A(x, t \mid y(\tau), \tau)
$$

Note that (2.2) and (2.3) define the transition distribution function and the transition pdf of $X(t)$, respectively, in the presence of the absorbing boundary $S(t ; \tau)$ conditioned upon $X(\tau)=y(\tau)$.

\section{THEOREM 2.1. For all $t \geq \tau$ set}

$$
D(t)=W[y(t), u(t)], \quad Q(t, \tau)=D(t) D(\tau) \int_{\tau}^{t} \frac{d \vartheta}{D^{2}(\vartheta)}, \quad R(t, \tau)=\frac{D(t) D(\tau)}{Q(t, \tau)}
$$

and let

$$
\Delta_{1}(t ; \tau)=c_{1}^{2}+4 c_{2} e^{-4 R(t, \tau)}
$$

For a strongly symmetric process, if $c_{1}>0, c_{2} \in \mathbf{R}$ and $\lim _{t \rightarrow+\infty} \Delta_{1}(t ; \tau)>0$ then, for all $t \geq \tau$ one has

$$
\alpha(x, t \mid y(\tau), \tau)=f(x, t \mid y(\tau), \tau)+c_{1} \varphi_{1}(x, t) \frac{\partial \phi_{1}(x, t)}{\partial x} f\left[\phi_{1}(x, t), t \mid y(\tau), \tau\right]
$$

$$
+c_{2} \varphi_{2}(x, t) \frac{\partial \varphi_{2}(x, t)}{\partial x} f\left[\phi_{2}(x, t), t \mid y(\tau), \tau\right]
$$




$$
(x<S(t ; \tau), y(\tau)<u(\tau)) \text { or }(x>S(t ; \tau), y(\tau)>u(\tau))
$$

where $S(t ; \tau)$ is a continuous solution of the following equation:

$$
W[y(t), S(t ; \tau)]=D(t)-\frac{Q(t, \tau)}{2 D(\tau)} \ln \left[\frac{c_{1}+\sqrt{\Delta_{1}(t ; \tau)}}{2}\right] .
$$

Proof. Let $\alpha_{1}(x, t \mid y(\tau), \tau)$ be the right-hand-side of (2.6). Clearly, since (1.7) holds, the function $\alpha_{1}(x, t \mid y(\tau), \tau)$ satisfies the Fokker-Planck equation. Furthermore, making use of (1.13) and (1.23), $\alpha_{1}(x, t \mid y(\tau), \tau)$ can be written as

$$
\begin{aligned}
& \alpha_{1}(x, t \mid y(\tau), \tau) \\
= & f(x, t \mid y(\tau), \tau)\left[1-c_{1} \exp \left\{-\frac{2}{Q(t, \tau)} W[x, u(t)] W[y(\tau), u(\tau)]\right\}\right. \\
& \left.-c_{2} \exp \left\{-\frac{2}{Q(t, \tau)} W[x, v(t)] W[y(\tau), v(\tau)]\right\}\right] .
\end{aligned}
$$

Recalling (2.1), and making use of (1.9) and of the first equality of (1.21), from (2.8) we obtain:

$$
\begin{aligned}
& \alpha_{1}(x, t \mid y(\tau), \tau) \\
& =-f(x, t \mid y(\tau), \tau) e^{-4 R(t, \tau)}\left\{c_{2} e^{-4 U(x, t, \tau)}+\left[c_{1} e^{-2 U(x, t, \tau)}-1\right] e^{4 R(t, \tau)}\right\},
\end{aligned}
$$

where $D(t)$ and $Q(t, \tau)$ are defined in (2.4) and where we have set

$$
U(x, t, \tau)=\frac{D(\tau)}{Q(t, \tau)} W[x, u(t)]
$$

If (i) $c_{1} \in \mathbf{R}$ and $c_{2}>0$ or (ii) $c_{2}<0$ and $\lim _{t \rightarrow+\infty} \Delta_{1}(t ; \tau)>0$, relation (2.9) becomes:

$$
\begin{aligned}
& \alpha_{1}(x, t \mid y(\tau), \tau) \\
& =-c_{2} f(x, t \mid y(\tau), \tau) e^{-4 R(t, \tau)}\left[e^{-2 U(x, t, \tau)}-\frac{2}{c_{1}+\sqrt{\Delta_{1}(t ; \tau)}}\right] \\
& \quad \times\left[e^{-2 U(x, t, \tau)}-\frac{2}{c_{1}-\sqrt{\Delta_{1}(t ; \tau)}}\right] .
\end{aligned}
$$

In order for $\left.\alpha_{1}(x, t\} y(\tau), \tau\right)$ to be a transition pdf in the presence of an absorbing boundary $S(t ; \tau)$, the right-hand-side of (2.11) must be zero at $x=S(t ; \tau)$, non negative for all $x<S(t ; \tau)$ and $y(\tau)<\lim _{t \downarrow} \tau S(t ; \tau) \quad[x>S(t ; \tau)$ and $\left.y(\tau)>\lim _{t \downarrow} \tau S(t ; \tau)\right]$; finally, it must satisfy the initial delta-condition. It is easy to prove that all these conditions hold iff $c_{1}>0, c_{2} \in \mathbf{R}, \lim _{t \rightarrow+\infty} \Delta_{1}(t ; \tau)>0$ and $S(t ; \tau)$ is solution of $(2.7)$. 
We remark explicitly that the boundary $S(t ; \tau)$, the solution of $(2.7)$, is such that $\lim _{t} \downarrow \tau S(t ; \tau)=u(\tau)$. In other words, for a fixed initial time $\tau$, starting from the symmetry curves $u(t)$ and $y(t)$, an absorbing boundary $S(t ; \tau)$ has been obtained, which approaches $u(t)$ as $t$ approaches $\tau$. It should be noticed the simplicity of the transition pdf in the presence of the absorbing boundary $S(t ; \tau)$. Such an expression follows from (2.6) or (2.8) for the entire class of strongly symmetric processes.

We now define the first-passage time through $S(t ; \tau)$ :

$$
T= \begin{cases}\inf _{t \geq \tau}\{t: X(t)>S(t ; \tau)\}, & X(\tau)=y(\tau)<u(\tau) \\ \inf _{t \geq \tau}\{t: X(t)<S(t ; \tau)\}, & X(\tau)=y(\tau)>u(\tau) .\end{cases}
$$

Let

$$
g(t \mid y(\tau), \tau)=\frac{\partial}{\partial t} P\{T<t\}
$$

be its pdf. The following theorem indicates a closed form relation of the FPT pdf through the boundary $S(t ; \tau)$ in terms of the free transition pdf.

THEOREM 2.2. Under the assumptions of Theorem 2.1 one has:

$$
g(t \mid y(\tau), \tau)=\frac{2 \sqrt{\Delta_{1}(t ; \tau)}}{c_{1}+\sqrt{\Delta_{1}(t ; \tau)}} \sqrt{A_{2}[S(t ; \tau)]} \frac{|D(\tau)|}{Q(t, \tau)} f[S(t ; \tau), t \mid y(\tau), \tau]
$$

Proof. Let $y(\tau)<u(\tau)$ or equivalently $D(\tau)>0$. As is well known,

$$
\int_{\nu_{1}}^{S(t ; \tau)} d z \alpha(z, t \mid y(\tau), \tau)+\int_{\tau}^{t} d \vartheta g(\vartheta \mid y(\tau), \tau)=1
$$

Making then use of (1.7), (1.10) and (2.6), from (2.15) one has:

$$
\begin{aligned}
g(t \mid y(\tau), \tau)= & -\int_{\nu_{1}}^{S(t ; \tau)} d z \frac{\partial}{\partial t} \alpha(z, t \mid y(\tau), \tau) \\
= & -\int_{\nu_{1}}^{S(t ; \tau)} d z \frac{\partial}{\partial t}\{f(z, t \mid y(\tau), \tau) \\
& -c_{1} \varphi_{1}\left[\phi_{1}(y(\tau), \tau), \tau\right] f\left[z, t \mid \phi_{1}(y(\tau), \tau), \tau\right] \\
& \left.-c_{2} \varphi_{2}\left[\phi_{2}(y(\tau), \tau), \tau\right] f\left[z, t \mid \phi_{2}(y(\tau), \tau), \tau\right]\right\} .
\end{aligned}
$$

Recalling that the function $f$ satisfies Fokker-Planck equation (1.2), Equation (2.16) can be written as: 


$$
\begin{aligned}
g(t \mid y(\tau), \tau)= & \int_{\nu_{1}}^{S(t ; \tau)} d z \frac{\partial}{\partial z}\left[A_{1}(z) f_{0}-\frac{1}{2} \frac{\partial}{\partial z}\left(A_{2}(z) f_{0}\right)\right] \\
& -c_{1} \varphi_{1}\left[\phi_{1}(y(\tau), \tau), \tau\right] \int_{\nu_{1}}^{S(t ; \tau)} d z \frac{\partial}{\partial z}\left[A_{1}(z) f_{1}-\frac{1}{2} \frac{\partial}{\partial z}\left(A_{2}(z) f_{1}\right)\right] \\
& -c_{2} \varphi_{2}\left[\psi_{2}(y(\tau), \tau), \tau\right] \int_{\nu_{1}}^{S(t ; \tau)} d z \frac{\partial}{\partial z}\left[A_{1}(z) f_{2}-\frac{1}{2} \frac{\partial}{\partial z}\left(A_{2}(z) f_{2}\right)\right]
\end{aligned}
$$

where we have set:

$$
f_{0}=f(z, t \mid y(\tau), \tau), \quad f_{\imath}=f\left[z, t \mid \phi_{i}(y(\tau), \tau), \tau\right] \quad(i=1,2) .
$$

From (1.7), (1.10) and (1.13) one obtains:

$$
\varphi_{1}\left[\phi_{1}(y(\tau), \tau), \tau\right] f\left[x, t \mid \phi_{1}(y(\tau), \tau), \tau\right]=f(x, t \mid y(\tau), \tau) e^{-2 U(x, t, \tau)}
$$

$$
\varphi_{2}\left[\phi_{2}(y(\tau), \tau), \tau\right] f\left[x, t \mid \phi_{2}(y(\tau), \tau), \tau\right]=f(x, t \mid y(\tau), \tau) e^{-4 V(x, t, \tau)}
$$

where $U(x, t, \tau)$ is defined in (2.10) and

$$
V(x, t, \tau)=\frac{D(\tau)}{Q(t, \tau)} W[x, v(t)]
$$

Furthermore, from (2.7) we have:

(2.21a) $1-c_{1} \exp \{-2 U[S(t ; \tau), t, \tau]\}-c_{2} \exp \{-4 V[S(t ; \tau), t, \tau]\}=0$,

$$
\exp \{-2 U[S(t ; \tau), t, \tau]\}=\frac{2}{c_{1}+\sqrt{\Delta_{1}(t ; \tau)}} .
$$

Calculating the integrals in (2.17) and making use of (2.19) and (2.21), relation (2.14) follows. The case $y(\tau)>u(\tau)$ can be analysed in a similar way, with the proviso that Equation (2.15) is changed to

$$
\int_{S(t ; \tau)}^{\nu_{2}} d z \alpha(z, t \mid y(\tau), \tau)+\int_{\tau}^{t} d \vartheta g(\vartheta \mid y(\tau), \tau)=1
$$

\section{Two absorbing boundaries}

Let $y(t), u(t)$ and $v(t)$ be symmetry curves such that $v(t)<y(t)<u(t)$ for all $t \geq \tau$, where $\tau \geq t_{0}$ is a fixed instant. We denote by $\phi_{0}(x, t)$ and $\varphi_{0}(x, t)$ the symmetry functions corresponding to $y(t)$, by $\psi_{1}(x, t)$ and $\varphi_{1}(x, t)$ the symmetry functions for $u(t)$ and by $\phi_{2}(x, t)$ and $\varphi_{2}(x, t)$ those for $v(t)$. Furthermore, we assume that 


$$
v(t)=\phi_{0}[u(t), t] .
$$

Assumption (3.1) implies that the symmetry curve $v(t)$ is the "image" of the symmetry curve $u(t)$ in the "mirror" $y(t)$ via the symmetry function $\phi_{0}$. Hence, for all $t \geq \tau$ ( $\tau$ fixed) conditions $y(t)<x<u(t)$ are equivalent to $\phi_{0}[y(t), t]>$ $\phi_{0}(x, t)>\phi_{0}[u(t), t]$, i.e. $v(t)<\phi_{0}(x, t)<y(t)$.

Let us now assume $P\{X(\tau)=y(\tau)\}=1$. Furthermore, let $S_{1}(t ; \tau)$ and $S_{2}(t ; \tau)$ denote continuous functions of $t$, taking values in $I$ for all $t \geq \tau$, such that (i) $S_{1}(t ; \tau)<S_{2}(t ; \tau) \forall t \geq \tau$ and (ii) $\lim _{t \downarrow} \tau S_{1}(t ; \tau)<y(\tau)<\lim _{t \downarrow} \tau S_{2}(t ; \tau)$. For all $t \geq \tau$ and $x \in\left(S_{1}(t ; \tau), S_{2}(t ; \tau)\right)$ we consider the following functions:

$$
\begin{gathered}
B(x, t \mid y(\tau), \tau)=P\left\{X(t)<x ; S_{1}(\vartheta ; \tau)<X(\vartheta)\right. \\
\left.<S_{2}(\vartheta ; \tau) \forall \vartheta \in(\tau, t) \mid X(\tau)=y(\tau)\right\}
\end{gathered}
$$

and

$$
\beta(x, t \mid y(\tau), \tau)=\frac{\partial}{\partial x} B(x, t \mid y(\tau), \tau)
$$

Note that (3.2) and (3.3) define the transition distribution function and the transition pdf of $X(t)$, respectively, in the presence of the absorbing boundaries $S_{1}(t ; \tau)$ and $S_{2}(t ; \tau)$.

Theorem 3.1. For all $t \geq \tau$ let $D(t), Q(t, \tau)$ and $R(t, \tau)$ be defined as in (2.4) and let

$$
\Delta_{2}(t ; \tau)=1-4 c_{1} c_{2} e^{-4 R(t, \tau)} .
$$

For a strongly symmetric process, if $c_{1}>0, c_{2}>0$ and $\lim _{t \rightarrow+\infty} \Delta_{2}(t ; \tau)>0$ then, for all $t \geq \tau$, one has

$$
\begin{aligned}
& \beta(x, t \mid y(\tau), \tau)=f(x, t \mid y(\tau), \tau) \\
& +c_{1} \varphi_{1}(x, t) \frac{\partial \psi_{1}(x, t)}{\partial x} f\left[\phi_{1}(x, t), t \mid y(\tau), \tau\right] \\
& +c_{2} \varphi_{2}(x, t) \frac{\partial \psi_{2}(x, t)}{\partial x} f\left[\phi_{2}(x, t), t \mid y(\tau), \tau\right] \\
& \quad\left(S_{1}(t ; \tau)<x<S_{2}(t ; \tau)\right),
\end{aligned}
$$

where $S_{i}(t ; \tau)(i=1,2)$ is a continuous function, solution of the following equation:

$$
W\left[y(t), S_{i}(t ; \tau)\right]=(-1)^{i}\left\{D(t)-\frac{Q(t, \tau)}{2 D(\tau)} \ln \left[\frac{1+\sqrt{\Delta_{2}(t ; \tau)}}{2 c_{3-i}}\right]\right\} .
$$


Proof. Let us denote by $\beta_{1}(x, t \mid y(\tau), \tau)$ the right-hand-side of (3.5). Clearly, since (1.7) holds, $\beta_{1}(x, t \mid y(\tau), \tau)$ satisfies the Fokker-Planck equation. Furthermore, by virtue of (1.13) and (1.23), $\beta_{1}(x, t \mid y(\tau), \tau)$ can be written as:

$$
\beta_{1}(x, t \mid y(\tau), \tau)=f(x, t \mid y(\tau), \tau)\left[1-c_{1} e^{-2 U(x, t, \tau)}-c_{2} e^{2 V(x, t, \tau)}\right] .
$$

Recalling (3.1) and making use of (1.9) and of the first of (1.21), from (3.7) we obtain:

(3.8) $\beta_{1}(x, t \mid y(\tau), \tau)=-f(x, t \mid y(\tau), \tau) e^{2 U(x, t, \tau)}\left[c_{1} e^{-4 U(x, t, \tau)}-e^{-2 U(x, t, \tau)}+c_{2} e^{-4 R(t, \tau)}\right]$.

If (i) $c_{1} c_{2}<0$ or (ii) $c_{1} c_{2}>0$ and $\lim _{t \rightarrow+\infty} \Delta_{2}(t ; \tau)>0$, relation (3.8) becomes:

$$
\begin{aligned}
& \beta_{1}(x, t \mid y(\tau), \tau) \\
& =-c_{1} f(x, t \mid y(\tau), \tau) e^{2 U(x, t, \tau)}\left[e^{-2 U(x, t, \tau)}-\frac{1+\sqrt{\Delta_{2}(t ; \tau)}}{2 c_{1}}\right] \\
& \quad \times\left[e^{-2 U(x, t, \tau)}-\frac{1-\sqrt{\Delta_{2}(t ; \tau)}}{2 c_{1}}\right] .
\end{aligned}
$$

For $\beta_{1}(x, t \mid y(\tau), \tau)$ to be a transition pdf in the presence of the absorbing boundaries $S_{t}(t ; \tau)(i=1,2)$, for all $t>\tau$ the right-hand-side of (3.9) must be zero at $x=S_{\imath}(t ; \tau) \quad(i=1,2)$, non negative for all $x \in\left(S_{1}(t ; \tau), S_{2}(t ; \tau)\right)$ and $y(\tau) \in\left(\lim _{t \downarrow} \tau S_{1}(t ; \tau), \lim _{t \downarrow} \tau S_{2}(t ; \tau)\right)$; furthermore, it must satisfy the initial delta-condition. It is easy to prove that all these conditions are satisfied iff $c_{1}>$ $0, c_{2}>0, \lim _{t \rightarrow+\infty} \Delta_{2}(t ; \tau)>0$ and each $S_{i}(t ; \tau)(i=1,2)$ is a solution of (3.6).

It is worth remarking explicitly that the solutions $S_{1}(t ; \tau)$ and $S_{2}(t ; \tau)$ of equations (3.6) tend to $v(\tau)$ and $u(\tau)$, respectively, as $t \downarrow \tau$.

We now define the first-passage time through $S_{1}(t ; \tau)$ or $S_{2}(t ; \tau)$, namely the first-exit time of the process from the region $\left(S_{1}(t ; \tau), S_{2}(t ; \tau)\right)$ :

$$
\mathscr{T}=\inf _{t \geq \tau}\left\{t: X(t) \notin\left(S_{1}(t ; \tau), S_{2}(t ; \tau)\right)\right\}, \quad X(\tau)=y(\tau)
$$

Let

$$
\gamma(t \mid y(\tau), \tau)=\frac{\partial}{\partial t} P\{\mathscr{T}<t\}
$$

be the pdf of $\mathscr{T}$. The following theorem shows how the first-passage time pdf through $S_{1}(t ; \tau)$ or $S_{2}(t ; \tau)$ can be obtained in closed form in terms of the free transition pdf. 
THEOREM 3.2. Under the assumptions of Theorem 3.1 one has:

$$
\begin{aligned}
& r(t \mid y(\tau), \tau) \\
& \quad=\frac{\sqrt{\Delta_{2}(t ; \tau)} D(\tau)}{Q(t, \tau)}\left\{\sqrt{A_{2}\left[S_{1}(t ; \tau)\right]} f\left[S_{1}(t ; \tau), t \mid y(\tau), \tau\right]\right. \\
& \left.\quad+\sqrt{A_{2}\left[S_{2}(t ; \tau)\right]} f\left[S_{2}(t ; \tau), t \mid y(\tau), \tau\right]\right\} .
\end{aligned}
$$

Proof. Since

$$
\int_{S_{1}(t ; \tau)}^{S_{2}(t ; \tau)} d z \beta(z, t \mid y(\tau), \tau)+\int_{\tau}^{t} d \vartheta \gamma(\vartheta \mid y(\tau), \tau)=1
$$

the proof goes as for Theorem 2.2 with the proviso that Equations (2.19) are now replaced by

$$
\begin{aligned}
& 1-c_{1} \exp \left\{-2 U\left[S_{i}(t ; \tau), t, \tau\right]\right\}-c_{2} \exp \left\{2 V\left[S_{i}(t ; \tau), t, \tau\right]\right\}=0 \\
& \quad(i=1,2) \\
& \exp \left\{-2 U\left[S_{i}(t ; \tau), t, \tau\right]\right\}=\frac{1+(-1) \sqrt{\Delta_{2}(t ; \tau)}}{2 c_{1}} \quad(i=1,2) .
\end{aligned}
$$

The results outlined in Sections 2 and 3 for transition and FPT pdf's hold for the entire class of strongly symmetric diffusion processes. Of course, for individual processes belonging to such a class also other types of problems may be posed and solved by ad hoc methods (see, for instance, Example (a) of Section 4).

\section{Examples}

The practical usefulness of the results of Sections 2 and 3 will now be pointed out via some non-trivial examples.

\subsection{Hyperbolic process}

Let

$$
\begin{gathered}
A_{1}(x)=\mu \frac{1-\lambda \exp \left\{-2 \mu x / \sigma^{2}\right\}}{1+\lambda \exp \left\{-2 \mu x / \sigma^{2}\right\}}, \quad A_{2}(x)=\sigma^{2}, \\
I=(-\infty,+\infty) \quad(\mu \in \mathbf{R}, \lambda \geq 0, \sigma>0) .
\end{gathered}
$$

Note that if $\lambda=0$ the hyperbolic process yields the Wiener process with drift $\mu$. The transition pdf is given by 


$$
\begin{aligned}
f(x, t \mid y, \tau)= & \frac{1+\lambda \exp \left\{-2 \mu x / \sigma^{2}\right\}}{1+\lambda \exp \left\{-2 \mu y / \sigma^{2}\right\}} \frac{1}{\sqrt{2 \pi \sigma^{2}(t-\tau)}} \\
& \times \exp \left\{-\frac{[x-y-\mu(t-\tau)]^{2}}{2 \sigma^{2}(t-\tau)}\right\} .
\end{aligned}
$$

This is seen to fulfill the symmetry condition (1.7) of Lemma 1.1 iff

$$
r(t)=a^{*} t+b^{*} \quad\left(a^{*}, b^{*} \in \mathbf{R}\right),
$$

$$
\phi(x, t)=2 r(t)-x
$$

$$
\varphi(x, t)=\frac{1+\lambda \exp \left\{-2 \mu x / \sigma^{2}\right\}}{1+\lambda \exp \left\{-2 \mu[2 r(t)-x] / \sigma^{2}\right\}} \exp \left\{-\frac{2\left(\mu-a^{*}\right)}{\sigma^{2}}[r(t)-x]\right\} .
$$

Furthermore, it satisfies relation (1.13) of Theorem 1.1 with

$$
Q(t, \tau)=t-\tau
$$

Hence, the hyperbolic process is strongly symmetric in the sense of Definition 1.1. i) One absorbing boundary. The symmetry curves are (cf. Section 2):

$$
y(t)=c t+d, \quad u(t)=a t+b, \quad v(t)=2 u(t)-y(t)
$$

with $a, b, c, d \in \mathbf{R}$ and $y(t)<u(t)<v(t)[y(t)>u(t)>v(t)]$ for all $t \geq \tau$. From (2.7) the absorbing boundary follows:

$$
S(t ; \tau)=u(t)-\frac{\sigma^{2}(t-\tau)}{2[u(\tau)-y(\tau)]} \ln \left[\frac{c_{1}+\sqrt{\Delta_{1}(t ; \tau)}}{2}\right]
$$

where

$$
\Delta_{1}(t ; \tau)=c_{1}^{2}+4 c_{2} \exp \left\{-\frac{4[u(t)-y(t)][u(\tau)-y(\tau)]}{\sigma^{2}(t-\tau)}\right\} .
$$

Hence, if $c_{1}>0, c_{2} \in \mathbf{R}$ and $\lim _{t \rightarrow+\infty} \Delta_{1}(t ; \tau)>0$, from (2.6) (or equivalently from (2.8)) and (2.14) the transition pdf in the presence of absorbing boundary (4.5) and the first-passage-time density through such boundary are obtained.

Note that for $\mu=0, \tau=0$ and $y(\tau)=0$ the boundary defined by (4.5) yields the well-known Daniels' result [6] for the standard Wiener process.

ii) Two absorbing boundaries. The symmetry curves are (cf. Section 3):

$$
y(t)=c t+d, \quad u(t)=a t+b, \quad v(t)=2 y(t)-u(t),
$$

with $a, b, c, d \in \mathbf{R}$ and $v(t)<y(t)<u(t)$ for all $t \geq \tau$. From (3.6) we obtain the absorbing boundaries: 


$$
\begin{aligned}
& S_{1}(t ; \tau)=v(t)-\frac{\sigma^{2}(t-\tau)}{2[u(\tau)-y(\tau)]} \ln \left[\frac{1+\sqrt{\Delta_{2}(t ; \tau)}}{2 c_{2}}\right] \\
& S_{2}(t ; \tau)=u(t)+\frac{\sigma^{2}(t-\tau)}{2[u(\tau)-y(\tau)]} \ln \left[\frac{1+\sqrt{\Delta_{2}(t ; \tau)}}{2 c_{1}}\right],
\end{aligned}
$$

where

$$
\Delta_{2}(t ; \tau)=1-4 c_{1} c_{2} \exp \left\{-\frac{4[u(t)-y(t)][u(\tau)-y(\tau)]}{\sigma^{2}(t-\tau)}\right\} .
$$

Hence, if $c_{1}>0, c_{2}>0$ and $\lim _{t \rightarrow+\infty} \Delta_{2}(t, \tau)>0$, from (3.5) (or equivalently from (3.7)) and (3.12) the transition pdf's in the presence of the absorbing boundaries (4.8) and the first-passage-time density through $S_{1}(t ; \tau)$ or $S_{2}(t ; \tau)$ are obtained.

It should be pointed out that the symmetry properties (1.7) and (1.13) of the transition pdf also appear in problems requiring infinite superpositions of symmetry curves, such as the hyperbolic process with the two linear absorbing boundaries $S_{1}(t)=c t+d_{1}, S_{2}(t)=c t+d_{2}\left(d_{1}<d<d_{2}\right)$ with $c$ and $d$ specifying the symmetry curve $y(t)$ in (4.7). Indeed, assuming again $P\{X(\tau)=y(\tau)\}=$ 1 , let us consider the doubly infinite system of linear symmetry curves

$$
\begin{array}{ll}
u_{n}(t)=c t+d+n\left(d_{2}-d_{1}\right) & n=0, \pm 1, \pm 2, \ldots \\
v_{n}(t)=c t+d_{2}-n\left(d_{2}-d_{1}\right) & n=0, \pm 1, \pm 2, \ldots
\end{array}
$$

Further, let us denote by $\phi_{1 n}(x, t)$ and $\varphi_{1 n}(x, t)$ the symmetry functions associated to $u_{n}(t) \quad(n=0, \pm 1, \pm 2, \ldots)$ and by $\phi_{2 n}(x, t)$ and $\varphi_{2 n}(x, t)$ those associated to $v_{n}(t) \quad(n=0, \pm 1, \pm 2, \ldots)$. From (4.2) one clearly obtains

$$
\begin{aligned}
& \phi_{1 n}(x, t)=2 u_{n}(t)-x, \\
& \begin{aligned}
\varphi_{1 n}(x, t)=\frac{1+\lambda \exp \left\{-2 \mu x / \sigma^{2}\right\}}{1+\lambda \exp \left\{-2 \mu\left[2 u_{n}(t)-x\right] / \sigma^{2}\right\}} \\
\times \exp \left\{-\frac{2(\mu-c)}{\sigma^{2}}\left[u_{n}(t)-x\right]\right\},
\end{aligned}
\end{aligned}
$$

$$
\begin{aligned}
& \phi_{2 n}(x, t)=2 v_{n}(t)-x, \\
& \begin{aligned}
& \varphi_{2 n}(x, t)= \frac{1+\lambda \exp \left\{-2 \mu x / \sigma^{2}\right\}}{1+\lambda \exp \left\{-2 \mu\left[2 v_{n}(t)-x\right] / \sigma^{2}\right\}} \\
& \times \exp \left\{-\frac{2(\mu-c)}{\sigma^{2}}\left[v_{n}(t)-x\right]\right\} .
\end{aligned}
\end{aligned}
$$

The transition pdf in the presence of the absorbing boundaries $S_{1}(t)$ and $S_{2}(t)$ is 
then given by

$$
\begin{aligned}
\beta(x, t \mid y(\tau), \tau) & \\
=\sum_{n=-\infty}^{+\infty} & \left\{\varphi_{1 n}(x, t)\left|\frac{\partial \phi_{1 n}(x, t)}{\partial x}\right| f\left[\phi_{1 n}(x, t), t \mid y(\tau), \tau\right]\right. \\
& \left.\quad-\varphi_{2 n}(x, t)\left|\frac{\partial \phi_{2 n}(x, t)}{\partial x}\right| f\left[\phi_{2 n}(x, t), t \mid y(\tau), \tau\right]\right\},
\end{aligned}
$$

which, by virtue of (1.13) and (4.3), can also be written as

$$
\begin{aligned}
\beta(x, t \mid y(\tau), \tau) & \\
=f(x, t \mid y(\tau), \tau) \sum_{n=-\infty}^{+\infty}[ & \exp \left\{-\frac{2}{\sigma^{2}(t-\tau)}\left[u_{n}(t)-x\right]\left[u_{n}(\tau)-y(\tau)\right]\right\} \\
& \left.\quad-\exp \left\{-\frac{2}{\sigma^{2}(t-\tau)}\left[v_{n}(t)-x\right]\left[v_{n}(\tau)-y(\tau)\right]\right\}\right],
\end{aligned}
$$

with $f$ defined by (4.1). To prove it, we remark that the series in (4.12) and (4.13) are absolutely convergent and term by term differentiable. Because of (1.7), from (4.12) one sees that $\beta(x, t \mid y(\tau), \tau)$ satisfies the Fokker-Planck equation (1.2). Moreover, from (1.7) and (1.10) we obtain

$$
\begin{aligned}
& \lim _{t \downarrow \tau}\left\{\varphi_{1 n}(x, t)\left|\frac{\partial \psi_{1 n}(x, t)}{\partial x}\right| f\left[\phi_{1 n}(x, t), t \mid y(\tau), \tau\right]\right\}= \begin{cases}\delta[x-y(\tau)] & n=0, \\
0 & n= \pm 1, \pm 2, \ldots,\end{cases} \\
& \lim _{t \downarrow \tau}\left\{\varphi_{2 n}(x, t)\left|\frac{\partial \psi_{2 n}(x, t)}{\partial x}\right| f\left[\phi_{2 n}(x, t), t \mid y(\tau), \tau\right]\right\}=0 \quad n=0, \pm 1, \pm 2, \ldots
\end{aligned}
$$

The right-hand side of (4.12) is immediately seen to satisfy the initial delta-condition. Finally, use of (4.13) shows that the absorption conditions on the boundaries are satisfied, i.e. $\beta\left[S_{1}(t), t \mid y(\tau), \tau\right]=\beta\left[S_{2}(t), t \mid y(\tau), \tau\right]=0$.

Note that for $\lambda=c=0$ expression (4.12) yields the well-known transition pdf for the Wiener process in the presence of two constant absorbing boundaries [5].

\subsection{Ornstein-Uhlenbeck process}

Let

$$
A_{1}(x)=\lambda x+\mu, \quad A_{2}(x)=\sigma^{2}, \quad I=(-\infty,+\infty) \quad(\lambda, \mu \in \mathbf{R}, \lambda \neq 0, \sigma>0) .
$$

The transition pdf of $X(t)$ is then:

$$
f(x, t \mid y, \tau)=\sqrt{\frac{\lambda}{\pi \sigma^{2}(\exp \{\lambda(t-\tau)\}-1)}}
$$




$$
\times \exp \left\{-\frac{\lambda[x+\mu / \lambda-(y+\mu / \lambda) \exp \{\lambda(t-\tau)\}]^{2}}{\sigma^{2}[\exp \{2 \lambda(t-\tau)\}-1]}\right\} .
$$

It satisfies the symmetry condition (1.7) iff

$$
\begin{aligned}
r(t) & =-\frac{\mu}{\lambda}+a^{*} e^{\lambda t}+b^{*} e^{-\lambda t} \quad\left(a^{*}, b^{*} \in \mathbf{R}\right) \\
\varphi(x, t) & =2 r(t)-x \\
\varphi(x, t) & =\exp \left\{-\frac{4 \lambda b^{*}}{\sigma^{2}} e^{-\lambda t}[r(t)-x]\right\} .
\end{aligned}
$$

Furthermore, it satisfies relation (1.13) of Theorem 1.1 with

$$
Q(t, \tau)=e^{-\lambda(t-\tau)} \frac{e^{2 \lambda(t-\tau)}-1}{2 \lambda} .
$$

This diffusion process is thus strongly symmetric.

i) One absorbing boundary. Following Section 2, the symmetry curves are seen to be

$$
y(t)=-\frac{\mu}{\lambda}+c e^{\lambda t}+d e^{-\lambda t}, \quad u(t)=-\frac{\mu}{\lambda}+a e^{\lambda t}+b e^{-\lambda t}, \quad v(t)=2 u(t)-y(t)
$$

with $a, b, c, d \in \mathbf{R}$ and $y(t)<u(t)<v(t)[y(t)>u(t)>v(t)]$ for all $t \geq \tau$. From (2.7) the absorbing boundary follows:

$$
S(t ; \tau)=u(t)-\frac{\sigma^{2} \exp \{-\lambda(t-\tau)\}[\exp \{2 \lambda(t-\tau)\}-1]}{4 \lambda[u(\tau)-y(\tau)]} \ln \left[\frac{c_{1}+\sqrt{\Delta_{1}(t ; \tau)}}{2}\right]
$$

where

$$
\Delta_{1}(t, \tau)=c_{1}^{2}+4 c_{2} \exp \left\{-\frac{8 \lambda \exp \{\lambda(t-\tau)\}[u(t)-y(t)][u(\tau)-y(\tau)]}{\sigma^{2}[\exp \{2 \lambda(t-\tau)\}-1]}\right\}
$$

Hence, if $c_{1}>0, c_{2} \in \mathbf{R}$ and $\lim _{t \rightarrow+\infty} \Delta_{1}(t ; \tau)>0$, from (2.6) (or equivalently from (2.8)) and (2.14) we obtain the transition pdf in the presence of the absorbing boundary (4.5) and the first-passage-time density through such boundary.

ii) Two absorbing boundaries. The symmetry curves now are:

$$
y(t)=-\frac{\mu}{\lambda}+c e^{\lambda t}+d e^{-\lambda t}, \quad u(t)=-\frac{\mu}{\lambda}+a e^{\lambda t}+b e^{-\lambda t}, \quad v(t)=2 y(t)-u(t)
$$

with $a, b, c, d \in \mathbf{R}$ and $v(t)<y(t)<u(t)$ for all $t \geq \tau$. The two absorbing boundaries then follow from (3.6): 
(4.21a)

$$
\begin{aligned}
& S_{1}(t ; \tau)=v(t)-\frac{\sigma^{2} \exp \{-\lambda(t-\tau)\}[\exp \{2 \lambda(t-\tau)\}-1]}{4 \lambda[u(\tau)-y(\tau)]} \ln \left[\frac{1+\sqrt{\Delta_{2}(t ; \tau)}}{2 c_{2}}\right] \\
& S_{2}(t ; \tau)=u(t)+\frac{\sigma^{2} \exp \{-\lambda(t-\tau)\}[\exp \{2 \lambda(t-\tau)\}-1]}{4 \lambda[u(\tau)-y(\tau)]} \ln \left[\frac{1+\sqrt{\Delta_{2}(t ; \tau)}}{2 c_{1}}\right]
\end{aligned}
$$

where

$$
\Delta_{2}(t, \tau)=1-4 c_{1} c_{2} \exp \left\{-\frac{8 \lambda \exp \{\lambda(t-\tau)\}[u(t)-y(t)][u(\tau)-y(\tau)]}{\sigma^{2}[\exp \{2 \lambda(t-\tau)\}-1]}\right\} .
$$

If $c_{1}>0, c_{2}>0$ and $\lim _{t \rightarrow+\infty} \Delta_{2}(t, \tau)>0$, from (3.5) (or equivalently from (3.7)) and (3.12) we then obtain the transition pdf in the presence of the two absorbing boundaries (4.8) and the first-exit-time density from $\left(S_{1}(t ; \tau), S_{2}(t ; \tau)\right)$.

Acknowledgments. We express our thanks to Professor Henry E. Daniels for his comments on an earlier version of this paper and for providing us with Reference [7].

\section{REFERENCES}

[1] Abrahams, J., A survey of recent progress on level-crossing problems for random processes, In Communications and Networks, A Survey of Recent Advances (I.F. Blake and H. V. Poor, eds.), (1986) 6-25. Springer-Verlag, New York.

[2] Blake, I. F. and Lindsey, W. C., Level-crossing problems for random processes, IEEE Trans. Inform. Theory, IT-19 (1973), 295-315.

[3] Buonocore, A., Giorno, V., Nobile, A.G. and Ricciardi, L.M., On the two-boundary first-crossing-time problem for diffusion processes, J. Appl. Prob., 27 (1990), 102-114.

[4] Buonocore, A., Nobile, A.G. and Ricciardi, L.M., A new integral equation for the evaluation of first-passage-time probability densities, Adv. Appl. Prob., 19 (1987), 784-800.

[5] Cox, D. R. and Miller, H.D., The Theory of Stochastic Processes, Chapman and Hall, London., (1965).

[6] Daniels, H.E., The minimun of a stationary Markov process superimposed on a U-shaped trend, J. Appl. Prob., 6 (1969), 399-408.

[7] Daniels, H.E., Sequential tests constructed from images, Ann. Stat., 10, N. 2 (1982), 394-400.

[8] Feller, W., The parabolic differential equations and the associated semi-groups of transformations, Ann. Math., 55 (1952), 468-518.

[9] Feller, W., Diffusion processes in one dimension, Trans. Amer. Math. Soc., 77 (1954), $1-31$.

[10] Giorno, V., Nobile, A.G. and Ricciardi, L.M., A symmetry-based constructive approach to probability densities for one-dimensional diffusion processes, J. Appl. Prob., 27 (1989), $707-721$.

[11] Giorno, V., Nobile, A.G., Ricciardi, L.M. and Sato, S., On the evaluation of firstpassage-time probability densities via non-singular integral equations, Adv. Appl. Prob., 21 (1989), 20-36. 


\author{
A. Di Crescenzo and L. M. Ricciardi \\ Dipartimento di Matematica e Applicazioni \\ University of Naples "Federico II" \\ via Cintia-80126 Naples, Italy \\ V. Giorno and A. G. Nobile \\ Dipartimento di Informatica e Applicazioni \\ University of Salemo \\ via S. Allende-84081 Baromissi (SA), Italy
}

